\title{
Das Publikum von Politikjournalisten als Interaktionspartner auf Twitter: Interaktionsstruktur, Motive und Erfahrungen anschreibender Nutzer
}

\author{
Christian Nuernbergk
}

Online publiziert: 23. Juni 2020

(C) Der/die Autor(en) 2020

Zusammenfassung Auf Twitter sind viele Journalisten mit persönlichen Accounts präsent und damit ein potenzieller Interaktionspartner für das Publikum. Untersuchungen aktiver Twitterer zeigen, dass auf der Netzwerkplattform politisch interessierte, persönlichkeitsstarke Nutzer interagieren, die sich vom Durchschnitt typischer Internetnutzer unterscheiden. Nachrichtenjournalisten berichten über negative Erfahrungen im direkten Publikumskontakt auf Twitter. Anders als bei Nutzerkommentaren auf Nachrichtenseiten sind öffentliche Anschriebe und Kontakte zu Journalisten auch unabhängig von einem Beitrag möglich. Zu diesem Phänomen existieren bislang jedoch kaum Studien. Über ein Tracking der Anschriebe wird daher erstens untersucht, wie häufig Politikjournalisten 2017 überhaupt in Tweets erwähnt wurden. Zweitens wurden die Nutzer befragt, die die Journalisten angeschrieben haben. Eine Nutzergruppe aus mehreren zehntausend Accounts nimmt Adressierungen vor. Die Erwähnungen verteilen sich unter den Journalisten ungleich. Die Nutzung von Blogs als alternative Informationsquelle und starke politische Orientierungen erklären das Vorkommen häufiger Interaktionen. Als Hauptmotivationen für Interaktionen zeigen sich das Bedürfnis nach eigener Meinungsäußerung und Kritik sowie das Weitergeben von neuen Informationen und Feedback an Journalisten. Sind diese Motivationen ausgeprägt, tendieren die Nutzer eher zu häufigen Interaktionen.

Prof. Dr. C. Nuernbergk ( $\square)$

Fachbereich II - Medienwissenschaft, Universität Trier, 54296 Trier, Deutschland

E-Mail: nuernbergk@uni-trier.de 


\title{
The audience of political journalists as interaction partners on Twitter: structures, motives and experiences of interactants
}

\begin{abstract}
Many journalists are present on Twitter with personal accounts. Twitter users are likely to directly interact with these journalists and to engage in a newsrelated discussion. So far, audience engagement research has mainly focused on user comments sections maintained by news outlets and ignored tweets directed to journalists. Through systematic tracking, this study investigates how often German political journalists received Twitter @mentions in 2017. In addition, those users who@mentioned the observed group of journalists were surveyed. Journalists were addressed by tens of thousands of accounts. However, @mentions were far from evenly distributed among the journalists. According to the survey, the main motivations for interactions are the need to express opinions and criticism as well as feedback motives such as passing on new information.
\end{abstract}

\section{Einleitung}

Das Feld der politischen Kommunikation unterliegt einem strukturellen Wandel, der vor allem durch das Internet und das Aufkommen sozialer Medien forciert wird. Die Veränderungen politischer Kommunikation sind vielschichtig (für eine Systematisierung siehe Dohle et al. 2014). Die Flexibilisierung des kommunikativen Zugangs und die Erweiterung von Partizipationsmöglichkeiten führen zu mehr Dynamik in der öffentlichen Medienkommunikation (Dohle et al. 2014; Neuberger 2018). Angenommen wird eine Hybridisierung des Mediensystems (Chadwick 2013), die auch auf die politische Kommunikation insgesamt zurückwirkt (z. B. Borucki und Jun 2018). Dies äußert sich beispielsweise in Medienrepertoires und Nutzungsstilen, die traditionelle Medien und Online-Medien integrieren, aber auch über gestiegene Vernetzungen und Bezugnahmen zwischen den Medienbereichen. Die Vernetzungsdynamiken können dazu führen, dass auch nicht-etablierte Angebote und Medienaktivisten episodisch einen hohen Grad an Sichtbarkeit erfahren können. Im hybriden Kommunikationsgefüge spielen Netzwerkplattformen eine wichtige Rolle. Insbesondere auf Twitter sind politische und mediale Akteure stark präsent. Für Bürger und Aktivisten, die über Medien Partizipation ausüben wollen, ist dies grundsätzlich ein interessantes Umfeld. In der vorliegenden Studie wird daher näher untersucht, mit welcher Motivation Twitter-Nutzer Journalisten anschreiben und aktiv Kontakt aufnehmen. Trotz der geringen Nutzung von Twitter in der Gesamtbevölkerung (Frees und Koch 2018; Hölig und Hasebrink 2018) haben Tweets sowie Twitter-Debatten mehrfach größere öffentliche Aufmerksamkeit erreicht. Journalisten, die Themen und Meinungen auf Twitter durch eigene Berichterstattung hervorheben, sind für eine entsprechende Verbreitung an ein größeres Publikum potenziell besonders relevant. Lobigs und Neuberger konstatieren angesichts der insgesamt schwierigeren Adressierbarkeit von Meinungsmacht im Internet, dass im Prinzip ,sämtliche Kommunikatoren und Anbieter in der öffentlichen Kommunikation“" in den Blick genommen werden sollten, soweit sie publizistische oder politische Relevanz besitzen (2018, S. 88). Ein Kennzeichen für die Relevanz der Kommunikatoren ist die Stabilität ihrer publizistischen 
Beteiligung, bzw. der Grad, mit dem ihnen die Fähigkeit zuerkannt wird, öffentliche Kommunikationswirkungen mit hoher Wahrscheinlichkeit zu erzielen (Lobigs und Neuberger 2018). Für den Journalismus im Internet, der in Deutschland bisher stark durch traditionelle Medienanbieter geprägt wird (Neuberger 2018), kann eine solche publizistische Beteiligung, aber auch die Zuschreibung von Einfluss auf der Publikumsseite angenommen werden (Albæk et al. 2014). Aufgrund ihrer publizistischen und politischen Relevanz wird vermutet, dass insbesondere Journalisten mit Rückmeldungen und Feedback aus dem Publikum rechnen müssen. Journalisten können auch von Nutzern angeschrieben werden, denen sie mitunter selbst gar nicht folgen. Die Rückmeldungen, die Journalisten und Medienorganisationen von einem Teil der Nutzer erhalten, geraten dabei zunehmend in Verruf (z. B. Hoffmann 2016; Reschke 2016). Da die Rückmeldungen selbst bereits plattform-öffentlich sind, kann hier nicht einfach von einer digitalen Variante eines Leserbriefs gesprochen werden. Nutzer, die journalistische Beiträge öffentlich kommentieren und Journalisten anschreiben, können auf diese Weise versuchen, episodisch Einfluss auf Diskurse auszuüben (Jungherr et al. 2019).

Aufbauend auf diesen Überlegungen will die vorliegende Studie durch eine Beobachtung der Twitter-Aktivitäten herausfinden, wie intensiv Journalisten Interaktionsversuchen ausgesetzt sind. Zweitens soll anhand einer Befragung ermittelt werden, welche Motivation hinter den öffentlichen Anschrieben von Journalisten steht. Denkbar ist, dass die nicht-journalistischen Interaktionspartner unterschiedliche Motive verfolgen und Teile von Ihnen nicht nur Feedback geben wollen oder Kritik an journalistischen Beiträgen üben, sondern auch darauf abzielen, über die Erwähnung und Einbindung von Journalisten stärker Aufmerksamkeit für ihre eigenen Anliegen zu erreichen. Ziel kann insofern eine indirekte Beeinflussung von Öffentlichkeit sein; im Sinn haben die Nutzer eher ein ,intendiertes Publikum“ und weniger die Journalisten als initiale Adressaten (Quandt 2018, S. 43). Diese Versuche der Meinungsbeeinflussung können im Extremfall bis hin zur gezielten Manipulation reichen (Thieltges und Hegelich 2017). Eine Besonderheit der Einbindung von Journalisten auf Twitter besteht darin, dass die @-Erwähnung auch unabhängig von einem journalistischen Beitrag erfolgen kann (anders als bei Nutzerkommentaren) und das potenzielle Publikum in dieser Interaktion nicht allein auf persönliche Follower limitiert ist, sondern beispielsweise durch Hashtags auch größere Nutzerkreise umfasst (Bruns und Moe 2014). Da viele Redaktionen die Möglichkeit des direkten Kommentierens auf ihren eigenen Webpräsenzen bereits beschränkt haben, stellen Plattformen wie Facebook und Twitter gewissermaßen Ausweichkanäle dar (Quandt 2018; Springer und Kümpel 2018).

\section{Theoretische Anknüpfungspunkte und Forschungsstand}

Im Zentrum dieser Studie stehen solche vom Publikum ausgehenden Interaktionen, die sich auf Journalisten beziehen. Der Wandel politischer Kommunikation ist natürlich weitgehender - das Aufkommen sozialer Medien betrifft das Verhältnis zwischen Politikern und Journalisten sowie zwischen Politikern und Publikum insgesamt (Broersma und Graham 2016). 
Im Forschungskontext der politisch-medialen Beziehungen wurde das Publikum bislang eher indirekt in die Betrachtungen miteinbezogen und vor allem das Verhältnis zwischen politischen Akteuren, ihren Sprechern und Journalisten analysiert (z. B. Pfetsch und Mayerhöffer 2011; Maurer und Beiler 2018). Die sozialen Medien erlauben allerdings nicht nur eine Ergänzung des üblichen kommunikativen Repertoires, mit dem Kontakte zwischen den Gruppen hergestellt werden, sondern liefern auch neue Möglichkeiten der Aufmerksamkeitserzeugung für politische Themen (Jungherr et al. 2019; Parmelee 2014). In der digitalen Öffentlichkeit erweitern sich die Beobachtungs- und Beeinflussungsmöglichkeiten auf der Publikumsseite. Die verstärkten Partizipationsmöglichkeiten in den sozialen Medien werden mittlerweile nicht nur als demokratisierendes Element betrachtet, sondern hinzukommend mit dem Erstarken des Populismus in den westlichen Demokratien in Zusammenhang gebracht (Jouët 2018; Quandt 2018). Wenn nicht-professionelle Kommunikatoren in den sozialen Medien Journalisten in ihren Beiträgen adressieren oder kontaktieren, handelt es sich allgemein um Phänomene der Online-Partizipation. Diese Beteiligung ist ambivalent einzuschätzen: Quandt (2018) hat mit seinem Konzept der ,dark participation“ prägnant darauf aufmerksam gemacht, dass die pauschale Annahme einer freiwilligen, hilfsbereiten und intrinsisch motivierten Partizipation zu kurz greift und Partizipation neben einer hellen eine dunkle, kalkulierende oder destruktive Seite haben kann.

Die auf dem Uses and Gratifications-Ansatz (Katz et al. 1973) aufbauende Forschung zum Kommentieren und Teilen von Medieninhalten hat entsprechend auch unterschiedliche Motivationen identifiziert (Kalogeropoulos et al. 2017; Ziegele 2016). Üblicherweise werden kognitive, affektive, sozial-integrative und identitätsbezogene Funktionen bzw. Motive unterschieden (Ziegele 2016). Nach Springer und Kümpel (2018) können auf die Gemeinschaft bezogene, sozial-interaktive Motive des Kommentierens von Nachrichten (z. B. Kontaktsuche, Meinungsaustausch) ana$\log$ auf Journalisten zielen. Als dominant erwiesen sich laut Ziegele et al. (2013) kognitive Motive, wie etwa das der Dissonanzreduktion. Diese kann durch Ergänzungen oder durch Korrekturen und Widerspruch erfolgen (Springer 2011; Ziegele et al. 2013). Dass Nutzer auf ihr Feedback eine Reaktion erhalten, ist allerdings unwahrscheinlich: Im Kommentarbereich beteiligen sich Journalisten nur höchst selten (Springer und Kümpel 2018). Für das Teilen von Nachrichten in sozialen Medien ermitteln Kümpel et al. (2015) auf Basis einer Studienübersicht in Teilen ähnliche Motivgruppen wie die Forschung zu Nutzerkommentaren: Unterschieden werden eigennützige Motive (Reputation, Status und Aufmerksamkeit erlangen), altruistische Motive (Informationen teilen) und sozial-interaktive Motive (Interagieren mit Anderen und soziale Zustimmung erhalten). Mit Blick auf die angesprochene „dark participation“ scheinen die beschriebenen Motivgruppen jedoch an Grenzen zu geraten. Sie müssten überdacht werden, um auch Phänomene wie ,Trolling“ oder die strategische Unterwanderung und Unterbrechung von politischen Diskussionen in den sozialen Medien zu erfassen. In solchen Versuchen wird ,eine weit verbreitete Einflussnahme auf den öffentlichen Meinungsaustausch“ erkannt (Thieltges und Hegelich 2017, S. 496). Infolgedessen ist es wahrscheinlich, dass Anschriebe im Umfeld politischer oder journalistischer Akteure auch mit manipulativen Absichten erfolgen können. Gerade herausgehobene Akteure wie Journalisten und Politiker 
gelten in der Risikoanalyse von Thieltges und Hegelich (2017) als gefährdet. Ebenso können koordinierte Formen der Desinformation auf Twitter auftreten (SchattoEckrodt et al. 2019).

Bislang wird in der Nutzungsforschung grundsätzlich analysiert, wie die Kommentierbereitschaft in den sozialen Medien und die Stärke politischer Orientierungen zusammenhängen. Auch der Einfluss des Medienvertrauens wird in neueren Studien betrachtet. Es konnte eine positive Assoziation zwischen der politischen OnlinePartizipation und der Stärke der Parteiidentifikation nachgewiesen werden (Valenzuela et al. 2012). Zu sozialen Medien belegen Kalogeropoulos et al. (2017) für Deutschland, Großbritannien, Italien, Spanien und die USA, dass die Häufigkeit des Kommentierens in sozialen Medien jeweils positiv mit der Stärke politischer Orientierungen assoziiert ist. Eine stärkere politische Orientierung wurde für Befragte angenommen, die sich auf einer Links-Rechts-Skala nah am linken oder rechten Pol verorten, also keine moderaten Einstellungen aufweisen. Der Zusammenhang zwischen Medienvertrauen und Kommentierhäufigkeit fällt dagegen negativ aus, d.h. dass eher Befragte mit niedrigerem Medienvertrauen zu einer häufigeren Beteiligung in den sozialen Medien tendieren (Fletcher und Park 2017). Befunde zu Nutzern mit medienfeindlichen Einstellungen zeigen, dass diese verstärkt zu Meinungsäußerungen im Netz neigen (Schindler et al. 2018). Zu den Kommentierenden auf Nachrichtenseiten lässt sich in Bezug auf die Soziodemographie und das Nachrichteninteresse außerdem festhalten, dass sie tendenziell älter, eher männlich und überdurchschnittlich stark an aktuellen Ereignissen interessiert sind. ${ }^{1}$ Für soziale Medien gilt dies nicht in gleicher Weise, denn hier partizipieren häufiger jüngere Nutzer - obschon nur eine Minderheit tatsächlich Kommentare zu Nachrichten verfasst (Schultz et al. 2017; Springer und Kümpel 2018). Jüngere Nutzer verfassen dabei eher Kommentare, ,um mit anderen zu diskutieren“ als ältere Nutzer (Schultz et al. 2017, S. 251).

Die Frage danach, ob zwischen den Kommentierenden auf Nachrichtenseiten und jenen Nutzern, die Journalisten öffentlich über soziale Medien anschreiben, Gemeinsamkeiten bestehen, lässt sich bislang schwer beantworten. Zu diesem spezifischen Verwendungsmuster fehlen aktuell dezidierte empirische Untersuchungen. Insbesondere auf Twitter sind viele Journalisten mit persönlichen Accounts präsent und damit potenzielle Interaktionspartner für das Publikum dort (Bruns 2018; Neuberger et al. 2014). Die Forschung konzentriert sich bislang größtenteils auf die journalistischen Kommunikatoren selbst und zeigt, dass Journalisten ihrerseits häufiger mit anderen Journalisten oder Politikern interagieren (Nuernbergk 2016; Hanusch und Nölleke 2019). Für die Interaktionen mit Nutzern gibt es abseits von Fallstudien weiterhin nur wenige quantitative Befunde. Allerdings zeigen Bruns und Nuernbergk (2019) mittels eines Vergleichs der Interaktionen zwischen Nutzern und deutschen sowie australischen Politikjournalisten, dass diese im Jahr 2017 insgesamt von ca. 117.000 unterschiedlichen Accounts (Deutschland) bzw. 231.000 Accounts (Australien) erwähnt oder retweetet werden. Demnach erhalten deutsche Politikjournalisten auch auf Twitter Rückmeldungen durch einen substanziellen Nutzerkreis. Wobei zu den

\footnotetext{
${ }^{1}$ Friemel und Dötsch (2015); Springer et al. (2015); Ziegele et al. (2013).
} 
Motiven der Nutzer, einzelne Journalisten direkt zu erwähnen, wie bereits akzentuiert, bislang weitere empirische Hinweise fehlen.

Höligs (2018) Vergleich von Internetnutzern mit aktiv twitternden Nutzern zeigt, dass letztere sich hinsichtlich ihrer Persönlichkeitsstärke, ihrer Soziodemographie und ihrer politischen Einstellungen vom Durchschnitt der Internetnutzer unterscheiden. Es lässt sich folglich vermuten, dass Journalisten einem besonders politisch interessierten, persönlichkeitsstarken Nutzertypus begegnen, wenn sie auf Twitter adressiert werden. Hölig (2018) zeigt für ,aktive Twitterer“ überdies, dass diese nicht nur eher von sich überzeugt sind, sondern auch eine Tendenz zum Narzissmus aufweisen, sowie extrovertierter und weniger ängstlich sind. Konfliktsituationen könnten dadurch wahrscheinlicher werden. Nachrichtenjournalisten berichten bei Publikumsanschrieben bereits über negative Erfahrungen auf Twitter (Binns 2017). Eine Befragung unter deutschen Journalisten ergab, dass zwei Fünftel der Befragten, Angriffe wie etwa Drohungen oder verbale Beleidigungen in Kommentaren oder über soziale Netzwerke erfahren haben (Preuß et al. 2017). Entsprechend dürften die Nutzerkontakte auch auf Twitter nicht selten konfliktbehaftet sein. In welchem Verhältnis jedoch kooperative, auf die Gemeinschaft zielende und empathische Tweets an Journalisten oder Politiker zu konfliktorientierten und negativ motivierten Anschrieben stehen, ist bisher ungeklärt.

Anders als in den Nutzerkommentar-Bereichen, können Nutzer auf Twitter die Journalisten auch außerhalb eines journalistischen Beitrags in einen Tweet einbinden. Zudem ist die @-Adressierung für die persönlichen Öffentlichkeiten der anschreibenden Nutzer sichtbar (Schmidt 2014). Somit ist denkbar, dass mit der direkt an Journalisten gerichteten Partizipationsform, indirekt ein weiteres Publikum intendiert ist, das beeinflusst werden soll. Aus der Kommentarforschung ist bereits bekannt, dass etwa jeder zehnte Kommentierer Kommentare im Internet verfasst, „um anderen zu zeigen, dass wir systematisch belogen werden“" (Schultz et al. 2017, S. 252). Fraglich ist, ob die Befunde der in diesem Abschnitt vorgestellten Kommentarforschung auch auf den vorliegenden Untersuchungskontext der direkten $\mathrm{Pu}-$ blikumsinteraktionen mit Journalisten übertragbar sind. Vor diesem Hintergrund soll der Beitrag drei Forschungsfragen klären:

FF1 Wie viele Nutzeraccounts schreiben Journalisten auf Twitter an und wie viele Interaktionen gehen von diesen Accounts aus?

FF2 Welche Erfahrungen machen Nutzer, die Journalisten anschreiben, und wie wahrscheinlich ist eine journalistische Rückmeldung auf ihre Beiträge?

FF3 Wie wirken sich die Interaktionsmotive, die Stärke der politischen Orientierung und die Persönlichkeitsstärke der Nutzer auf die Häufigkeit ihrer Interaktionen mit Journalisten aus? 


\section{Methodisches Vorgehen}

\subsection{Datenerhebung und Stichprobe}

Für die Studie wurden solche Tweets, die per @-Adressierung (,Mention“) an die Accounts von Politikjournalisten gerichtet sind, unter Verwendung des Twitter Capture and Analysis Toolkit (TCAT) seit Ende 2016 aufgezeichnet (siehe zum Toolkit Borra und Rieder 2014). Für das Tracking wurden für Journalisten in der Bundespressekonferenz (BPK) 2016/2017 zunächst 461 Accounts recherchiert und anschließend deren eigene Aktivitäten sowie die an sie gerichteten Tweets erfasst. Im Verein der BPK, der mehr als 900 Mitglieder umfasst, organisieren sich (Hauptstadt-)Journalisten, die über Bundespolitik aus Berlin oder Bonn berichten. Sie gehören bekannten Leitmedien (zur Einteilung Greck 2017), aber auch anderen publizistischen Einheiten, wie etwa Lokal- und Regionalzeitungen oder den Nachrichtensendungen privater Rundfunkprogramme und Landesrundfunkanstalten, an.

Im Jahr 2017 konnten insgesamt 548.651 Erwähnungen per @-Adressierung an die BPK-Journalisten (mit Twitter-Account) erfasst werden, die wiederum von 86.900 Accounts abgesetzt wurden (zur Funktionsweise der Operatoren auf Twitter siehe Thimm et al. 2012). Über das Tracking ließen sich somit auch Accounts ermitteln, die mit Journalisten öffentlich interagiert haben (FF1).

Diese Nutzergruppe wurde im Rahmen der Studie zusätzlich um Teilnahme an einer (Online-)Befragung gebeten, um FF2 und FF3 zu beantworten. Aus einer Gruppe von 12.018 Accounts, die im Zeitraum April/Mai 2017 mit den Journalisten interagierten, wurde eine Zufallsstichprobe von 4000 Accounts gezogen, wobei abzüglich institutioneller sowie gesperrter Accounts, 3743 Accounts in der Stichprobe verblieben sind. Die Onlinebefragung der Accountinhaber erfolgte im Juni 2017; via Einladungstweets wurde um Teilnahme an der Befragung gebeten. Eine Benachrichtigung darüber erhielten die Nutzer nur sofern sie in ihren persönlichen Einstellungen angegeben hatten, dass sie auch Accounts unabhängig von einem Folgeverhältnis anschreiben dürfen (standardmäßig deaktiviert). Ein massenhaftes Anschreiben mit identischen Tweets wird von Twitter durch plattformspezifische Filter unterbunden, so dass die Einladungstweets kleinschrittig und in mehreren Textvarianten von extra für die Befragung angelegten Accounts verschickt wurden. Trotz dieser Einschränkung nahmen 159 Personen an der Onlinebefragung teil (4,2\%), wobei Personen, die sich selbst als Journalisten identifizierten, ausgeschlossen wurden. Von den übrigen Befragten gaben 92,6\% $(n=136)$ an, mindestens selten über Twitter mit Journalisten zu interagieren.

\subsection{Untersuchungsdesign}

Der Fragebogen bestand aus mehreren Teilbereichen. Grundlegend für die Durchführung der Studie war zunächst die Ermittlung der Interaktionshäufigkeit auf Twitter. Die Häufigkeit der Interaktionen der Befragten mit Journalisten und anderen, getrennt aufgeführten Nutzertypen (Politiker, Prominente, andere Nutzer) wurde dafür vergleichend abgefragt. Da vermutet wurde, dass die Befragten die Häufigkeit bisheriger Interaktionen nicht exakt quantifizieren können, wurde eine Ordinalska- 
la gewählt $(1=($ noch $)$ nie $-5=$ sehr häufig $)$. Ebenso wurden weitere grundlegende Variablen, die sich speziell auf Twitter beziehen, erfragt. Erhoben wurde der Beginn der eigenen Mitgliedschaft (also das „Accountalter“). Auch die Häufigkeit der Verwendung von Twitter zum Verfassen eigener Beiträge sowie zum Lesen von Tweets wurde abgefragt (von ,seltener als monatlich“ bis ,mehrmals täglich“). Zu den ermittelten soziodemographischen Variablen zählen Alter, Geschlecht, formale Bildung und der Beschäftigungsstatus.

Die Bereiche zur Beantwortung von FF2 fokussieren insbesondere auf die Erfahrungen, die die anschreibenden Nutzer im Kontext ihrer Interaktionsversuche mit Journalisten machen. $\mathrm{Zu}$ den Erfahrungen mit Interaktionen wurde eine Liste mit neun Items erstellt, die den Austausch von Journalisten mit Nutzern charakterisieren sollen (z. B. Journalistinnen und Journalisten ,,... antworten auf Kritik von Nutzern“, „... ignorieren Nutzer, die sie anschreiben“). Die Nutzer wurden gebeten, auf einer fünfstufigen Skala anzugeben, wie stark diese Items, die vor allem auf die journalistische Rückmeldung eingehen, ihrer Erfahrung nach zutreffen $(1=$ trifft überhaupt nicht $\mathrm{zu}-5=$ trifft voll und ganz $\mathrm{zu}$ ). Eine weitere Frage mit zehn Items bezog sich wiederum darauf, wie die Nutzer die Häufigkeiten einzelner journalistischer Aktivitäten auf Twitter einschätzen (Antwortoptionen ,häufig“, „,selten“, ,nie“). Gefragt wurde hier auch nach Aktivitäten, die sich nicht auf Interaktionen mit dem Publikum beziehen (z. B. Journalistinnen und Journalisten ,... nutzen die Plattform privat", ,,... teilen dort ihre persönliche Meinung mit", ,,... werben dort für sich und ihre Arbeit“, ,,... diskutieren mit Politikerinnen und Politikern“").

Die Bereiche zur Beantwortung von FF3 bezogen sich sowohl auf die Motive Journalisten anzuschreiben als auch auf weitere Merkmale, die die Befragten anhand von Persönlichkeitsmerkmalen wie der Persönlichkeitsstärke charakterisieren und ihr politisch-mediales Profil herausarbeiten sollen (politische Orientierung, politisches Partizipationsverhalten, Mediennutzung und Medienvertrauen).

Die Motive zur Interaktion mit Journalisten auf Twitter wurden in Anlehnung an Ziegele et al. (2013) abgefragt (Motivgruppen: Informationsinteresse, Ergänzung fehlender Standpunkte, Korrektur, Widerspruch, Diskussion, Gemeinschaftsgefühl) und an den vorliegenden Kontext angepasst. Die Abfrage erfolgte anhand von fünfstufigen Skalen ( $1=$ trifft überhaupt nicht $\mathrm{zu}-5=$ trifft voll und ganz $\mathrm{zu})$.

Zur Mediennutzung der Befragten wurden das Medienrepertoire (Häufigkeit der Nutzung von Nachrichtenquellen) sowie ihr Medienvertrauen gemessen. Für die Abfrage des generalisierten Medienvertrauens wurde die Formulierung aus Hölig und Hasebrink (2016) übernommen (fünfstufige Skala mit Zustimmung zum Item „Ich glaube, man kann dem Großteil der Nachrichten meist vertrauen“, $M=2,90$; $S D=1,15)$ und das Vertrauen auch hinsichtlich einzelner Nachrichtenkanäle abgefragt. Die Befragten wurden überdies gebeten anzugeben, wie häufig sie bereits Nutzerkommentare auf Online-Nachrichtenseiten verfasst haben $(1=($ noch $)$ nie $5=$ sehr häufig).

Die politische Online-Partizipation wurde in Anlehnung an Gil de Zúñiga et al. (2012) mit acht Items gemessen, mit denen die Partizipationshäufigkeit in den letzten 12 Monaten, unter Verwendung einer fünfstufigen Skala, abgefragt wurde $(1=$ nie $5=$ sehr häufig, $M=2,62 ; S D=0,76$; Mittelwertindex). Mittels einer weiteren Frage wurden zwölf generelle politische Partizipationsformen (übernommen aus ALLBUS 
2008, siehe Gesis 2010), die schon einmal praktiziert wurden, ermittelt. Außerdem wurden die Befragten auf einer fünfstufigen Skala gebeten, ihr Interesse an Informationen über das politische Geschehen einzustufen ( 1 = überhaupt nicht interessiert $5=$ sehr interessiert, $M=4,74 ; S D=0,55)$. Die politische Orientierung wurde mit einer elfstufigen Links-Rechts-Skala im Anschluss an Vonbun und Schönbach (2014) erhoben, auf der die Befragten ihre politische Einstellung selbst einschätzen sollten ( $1=$ links $-11=$ rechts, $M=4,57 ; S D=2,44)$. Durch die Selbsteinschätzung lassen sich in der Analyse moderate und starke, zu den Extremen tendierende politische Orientierungen unterscheiden.

Zur Erfassung von Persönlichkeitsmerkmalen wurde das Konzept der Persönlichkeitsstärke übernommen, mit dem einflussreiche Personen mit Ausstrahlung und Durchsetzungskraft ermittelt werden (Noelle-Neumann 1986). In der vorliegenden Studie wurde die Persönlichkeitsstärke im Anschluss an Schenk und Scheiko (2011) mit zehn Items und fünffach gestuften Antwortmöglichkeiten ( $1=$ trifft überhaupt nicht zu $-5=$ trifft voll und ganz zu) gemessen. Für die Auswertung wurden die Items nach einem Schema ( $1=0$ Punkte, 2=0,25 Punkte, 3=0,5 Punkte, $4=0,75$ Punkte, 5=1 Punkt) gewichtet und ein Summenindex gebildet $(\alpha=0,83$; Min $=0$ bis $\operatorname{Max}=10 ; M=5,27 ; S D=0,93)$.

Die Datenauswertung für FF3 erfolgte mittels binär-logistischer Regression. Die ursprünglich ordinalskalierte AV zur Häufigkeit der Interaktionen wurde binarisiert. Die Reduktion der ursprünglichen Skala auf zwei Ausprägungen vor der Regression empfiehlt sich hier, weil bestimmte Ausprägungen sonst zu wenige Beobachtungen aufweisen. Aus diesem Grund wurde die Modellierung einer ordinal-logistischen Regression verworfen. Für die Regression wird hier speziell das Vorliegen einer mindestens häufigen Interaktion mit Journalisten vorhergesagt.

\section{Ergebnisse}

Anschließend an die Reihung der Forschungsfragen werden zunächst grundlegende Ergebnisse zur Häufigkeit der Interaktionen auf Basis des Trackings berichtet (zu FF1) und danach schließlich die Ergebnisse der Befragung präsentiert (zu FF2, FF3). Das Tracking der Journalisten und jener Tweets, in denen sie angeschrieben wurden, lief über den Befragungszeitraum hinaus bis zum Ende des Jahres 2017, weswegen sich die Angaben zur Häufigkeit der Interaktion, auf die Twitter-Aktivitäten während des gesamten Jahres beziehen.

Insgesamt wurden 400 aktiv twitternde Journalisten-Accounts ermittelt. Das entspricht $87 \%$ der identifizierten Accounts in der BPK. Im Mittel verfassten diese Accounts 609 Tweets pro Jahr $(M D=150)$ und haben 2436 Follower aufgewiesen $(M D=633)$. Die Twitter-Aktivität ist also nicht besonders ausgeprägt und beläuft sich durchschnittlich auf weniger als zwei Tweets pro Tag. Dennoch, so zeigt die Beantwortung von FF1, werden die beobachteten Journalisten im gleichen Zeitraum in 714.206 Tweets erwähnt oder retweetet. Insgesamt werden 548.651 Tweets erfasst, in denen @-Mentions von Journalisten enthalten sind. Diese werden von 86.900 Accounts abgesetzt. Im Mittel wird ein Journalist also von 583 Accounts pro Jahr auf diese Weise erwähnt $(M D=141)$ bzw. erhält durchschnittlich Hinweise zu 
1411 Tweets $(M D=224)$, in denen sein Nutzername erwähnt wird. Bei Differenzierung der Journalisten nach ihrer Verwendungshäufigkeit von Twitter, zeigt sich, dass das Dezil der Accounts mit den meisten Tweets $77 \%$ dieser Erwähnungen erhält. Somit verteilen sich die Nutzerinteraktionen sehr unterschiedlich auf die untersuchten Politikjournalisten. Während das Gros der Journalisten mit einer geringen Zahl an Nutzerinteraktionen pro Tag rechnen kann, erhalten die 40 aktivsten Journalisten eine deutliche größere Zahl an Rückmeldungen, nämlich über zwanzig Erwähnungen pro Tag (Jahreswerte: $M=8512 ; M D=4072$ ).

$\mathrm{Zu}$ den folgenden Befragungsergebnissen sollen zunächst einige strukturelle Befunde vorgestellt werden, bevor FF2 und FF3 aufgegriffen werden. Die Zusammensetzung der Befragten ähnelt in der Tendenz den Befunden von Hölig (2018) zu ,aktiven Twitterern“ in Deutschland. Zur Alters- und Geschlechtsstruktur der Personen, die auf Twitter mit Journalisten interagieren, ist jedoch anzumerken, dass der Anteil der Befragten mit männlichem Geschlecht $(82 \%, n=107)$ deutlich über dem in der Befragung von Hölig (2018, S. 152) liegt (,aktive Twitterer“: 62\%). Zudem sind die Befragten etwas älter $(M=42,1 ; n=105)$ als in der Vergleichsstudie. Es zeigt sich ein hoher formaler Bildungsgrad: Laut Selbstauskunft verfügt ein Anteil von $85 \%(n=101)$ über einen hohen Bildungsabschluss (also (Fach-)Hochschulreife oder abgeschlossenes Studium an einer Universität/Fachhochschule). Vier Fünftel der Befragten verlautbaren, an Informationen über das politische Geschehen sehr interessiert zu sein $(79 \%, n=115)$. Lediglich $18 \%$ der Befragten $(n=117)$ geben an, dass sie bisher (noch) nie Kommentare auf Online-Nachrichtenseiten verfasst haben. Häufig praktizieren dies $15 \%$ der Befragten, sehr häufig noch $9 \%$.

Betrachtet man die Häufigkeit der Interaktionen auf Twitter, bestätigen sich die Befunde des Trackings. Alle Befragten geben an, dass sie mindestens selten mit anderen Nutzern auf Twitter per @-Erwähnung interagieren. Journalisten schreiben $93 \%$ der Befragten mindestens selten an. ${ }^{2}$ Annähernd $26 \%$ interagieren mit Journalisten auf Twitter mindestens häufig (Tab. 1). Politiker auf Twitter haben interessanterweise mit gleicher Häufigkeit bereits mehr als $32 \%$ der Befragten angeschrieben. Bei sonstigen prominenten Akteuren wie Sportlern wird dagegen nur selten von der @-Adressierung Gebrauch gemacht, was wiederum dokumentiert, dass die Befragten vorwiegend am politisch-medialen Komplex interessiert sind.

Bevor auf die Erfahrungen zu den Interaktionen im Rahmen der Beantwortung von FF2 eingegangen wird, lässt sich anhand der Tracking-Daten verdeutlichen, dass ein Großteil der 86.900 Accounts, die Journalisten binnen des Jahres 2017 erwähnt haben, selbst keine Resonanz erfahren hat. Im gleichen Zeitraum richteten sich die beobachteten Journalisten in ihren Tweets an 19.751 Accounts per @-Mention. Nicht alle Nutzer können also mit Antworten rechnen; dennoch illustrieren diese Aufzeichnungen, dass Journalisten auf Twitter zusammengenommen durchaus mit einem größeren Nutzerkreis öffentlich interagieren. Über das Tracking kann allerdings nicht erfasst werden, ob die Journalisten die Nutzer möglicherweise über private Direktnachrichten angeschrieben haben.

\footnotetext{
2 Denkbar ist, dass die Befragten die Journalisten in einigen Fällen unwissentlich in ihren Tweets erwähnt haben und es deswegen zu kleineren Abweichungen gegenüber den Tracking-Befunden kommt.
} 
Tab. 1 Häufigkeit, mit der Journalisten und andere Akteursgruppen auf Twitter per @-Mention erwähnt werden (in \%, Befragung 2017, $n=136$ )

\begin{tabular}{lllll}
\hline & Journalisten & Politiker & $\begin{array}{l}\text { Prominente } \\
\text { (Sportler, Schauspieler etc.) }\end{array}$ & $\begin{array}{l}\text { Andere Nutzer } \\
\text { allgemein }\end{array}$ \\
\hline Sehr häufig & 4,4 & 4,4 & 1,5 & 33,8 \\
Häufig & 21,3 & 27,9 & 3,7 & 37,5 \\
Manchmal & 41,2 & 39,0 & 16,2 & 21,3 \\
Selten & 25,7 & 22,1 & 41,9 & 7,4 \\
(Noch) nie & 7,4 & 6,6 & 36,8 & 0 \\
\hline
\end{tabular}

Die Ergebnisse in Tab. 2 liefern Rückschlüsse, inwieweit die Befragten überhaupt Antworten von Journalisten wahrnehmen. So werden insgesamt eher Antworten auf solche Tweets wahrgenommen, die die Journalisten loben als auf jene Tweets, die sie kritisieren; die Zustimmungswerte sind aber überwiegend gering. Mehrheitlich abgelehnt werden die Statements: ,,antworten auf Kritik von Nutzern“, ,diskutieren

Tab. 2 Bewertungen von Aussagen zum Austausch von Journalisten mit Nutzern auf Twitter auf Basis eigener Erfahrungen (Mittelwerte, Befragung 2017)

\begin{tabular}{|c|c|c|c|c|c|c|}
\hline \multirow[t]{2}{*}{$\begin{array}{l}\text { Journalistinnen und } \\
\text { Journalisten ... }\end{array}$} & \multicolumn{2}{|c|}{$\begin{array}{l}\text { Mind. häufige Interaktion } \\
\text { mit Journalisten }\end{array}$} & \multicolumn{2}{|c|}{$\begin{array}{l}\text { Seltenere Interaktion mit } \\
\text { Journalisten }\end{array}$} & \multicolumn{2}{|c|}{ Gesamt } \\
\hline & $M$ & $S D$ & $M$ & $S D$ & $M$ & $S D$ \\
\hline $\begin{array}{l}\ldots \text { antworten auf Lob von } \\
\text { Nutzern }(U=707,0)^{*}\end{array}$ & 3,71 & 1,04 & 3,03 & 1,12 & 3,24 & 1,14 \\
\hline $\begin{array}{l}\text {... ignorieren Nutzer, die } \\
\text { sie anschreiben }\end{array}$ & 3,10 & 1,14 & 3,24 & 1,12 & 3,20 & 1,12 \\
\hline $\begin{array}{l}\text {... liefern Nutzern ergän- } \\
\text { zende Informationen }\end{array}$ & 3,19 & 1,20 & 3,06 & 1,17 & 3,10 & 1,17 \\
\hline $\begin{array}{l}\text {... erklären Sachverhalte, } \\
\text { wenn sie danach gefragt } \\
\text { werden }\end{array}$ & 3,25 & 1,30 & 2,93 & 1,05 & 3,03 & 1,14 \\
\hline $\begin{array}{l}\text {... widersprechen Nutzern, } \\
\text { die sie anschreiben }\end{array}$ & 3,19 & 0,97 & 2,96 & 1,09 & 3,03 & 1,05 \\
\hline $\begin{array}{l}\text {... antworten auf Kritik } \\
\text { von Nutzern }\end{array}$ & 3,06 & 1,11 & 2,81 & 1,17 & 2,89 & 1,16 \\
\hline $\begin{array}{l}\text {... diskutieren mit Nutzern } \\
\text { politische Themen }\end{array}$ & 3,09 & 1,17 & 2,78 & 1,18 & 2,88 & 1,18 \\
\hline $\begin{array}{l}\text {... geben Nutzern Feed- } \\
\text { back }\end{array}$ & 3,10 & 1,06 & 2,67 & 1,06 & 2,80 & 1,07 \\
\hline $\begin{array}{l}\text {... stimmen Nutzern zu, } \\
\text { die sie anschreiben }\end{array}$ & 2,73 & 0,87 & 2,42 & 1,01 & 2,52 & 0,97 \\
\hline
\end{tabular}

$n=96-105 ; 5$-Stufige Likert-Skala ( $1=$ trifft überhaupt nicht $\mathrm{zu}-5=$ trifft voll und ganz $\mathrm{zu})$

Mittelwertberechnung, Mann-Whitney-U-Test

Die Befragten werden in Gruppen verglichen: mind. häufige Interaktion (Antwort häufig/sehr häufig) vs. seltenere Interaktion (Antwort selten/manchmal).

Frage: „Wenn Sie an Journalistinnen und Journalisten und ihren Austausch mit Nutzern auf Twitter denken, inwieweit treffen die folgenden Aussagen Ihrer Erfahrung nach zu?“

Befragten, die ,(noch) nie“ Journalisten angeschrieben haben, wurde die Frage nicht gestellt. $* p<0,05$ 
mit Nutzern politische Themen“, ,geben Nutzern Feedback“ und „stimmen Nutzern zu, die sie anschreiben".

Gefragt nach ihren generellen Beobachtungen zu Journalisten auf Twitter, antworteten $77 \%(n=97)$ der Befragten, dass Journalisten dort häufig ihre persönliche Meinung mitteilen. $62 \%(n=100)$ gaben an, dass Journalisten auf Twitter häufig beschimpft oder angegriffen werden, was auf ein eher negatives Nutzerfeedback bzw. ein eher ,raues Klima“ hindeutet. Diskussionen von Journalisten mit anderen Journalisten werden von $47 \%(n=93)$ der Befragten häufig wahrgenommen. Dagegen registrieren die Befragten mit gleicher Häufigkeit seltener Diskussionen zwischen Journalisten und Politikern ( $40 \%, n=94)$ sowie Diskussionen zwischen Journalisten und Bürgern (32\%, $n=102)$.

$\mathrm{Zu}$ den Motiven der Nutzer, die Journalisten auf Twitter anschreiben, macht die Auswertung in Tab. 3 deutlich, dass durch das Anschreiben von Journalisten vor allem verschiedene kognitive Funktionen erfüllt werden und entsprechende Motive - wie etwa das Mitreden, das öffentliche Äußern von Meinungen oder das Hinweisen auf fehlende Standpunkte - die höchsten Zustimmungsgrade erfahren. Auch das Interesse am Thema erweist sich als ein wichtiger Treiber. Affektive Funktionen, wie das Empfinden von Spaß, erhalten dagegen niedrigere Zustimmungswerte. Einzelne sozial-integrative Funktionen, wie etwa der Aufbau von Sozialbeziehungen, wird nur teilweise zugestimmt (mit Journalisten in Kontakt treten), oder sie werden tendenziell abgelehnt (Gleichgesinnte finden).

Für weitere Berechnungen wurden mittels Hauptkomponenten-Analyse zunächst drei zentrale Motivfaktoren identifiziert und die auf Faktor 1 bzw. Faktor 2 ladenden Items jeweils zu einer Skala zusammengefasst, die wiederum akzeptable Reliabilitäten erzielen (Tab. 3).

Die beiden Motivfaktoren 1 und 2 zeigen unterschiedliche kognitive Funktionen des Anschreibens von Journalisten: Im ersten Fall geht es um das öffentliche Kritisieren und das Artikulieren eigener Standpunkte; im zweiten Fall um inhaltliche Unterstützung und Feedback, zum Beispiel durch Hinweise auf neue Informationen oder das Liefern von Korrekturvorschlägen. Der dritte Motivfaktor vereint mehrere Items, die insgesamt weniger stark auf die Journalisten selbst gerichtet sind als bei den ersten beiden Faktoren; sie gruppieren sich vor allem um das Interesse am behandelten Thema der Interaktion.

Abschließend soll geklärt werden, welche Merkmale mit einer häufig vorliegenden Interaktion der Befragten mit Journalisten auf Twitter zusammenhängen (FF3). Für die Auswertung im Rahmen einer binär-logistischen Regression, werden verschiedene Variablen herangezogen, darunter die gebildeten Motiv-Skalen „Kritik und Meinungsäußerung" sowie „Feedback und neue Informationen geben“ (siehe Tab. 3). Der Summenindex zur Persönlichkeitsstärke der Befragten (siehe Abschn. 3 zum Untersuchungsdesign), der Mittelwertindex zur politischen Online-Partizipation sowie die Stärke der politischen Orientierung (gruppiert nach moderat (4-8) und 
Tab. 3 Faktorladungen aus einer Hauptkomponentenanalyse mit Varimax Rotation für 11 Items, die Motive der Interaktion mit Journalisten auf Twitter messen (Befragung 2017)

\begin{tabular}{|c|c|c|c|c|}
\hline $\begin{array}{l}\text { Ich verfasse Tweets an } \\
\text { Journalistinnen und Journalisten, } \\
\text { weil/damit ... }\end{array}$ & $\begin{array}{l}\text { Gesamt } \\
M\end{array}$ & $\begin{array}{l}\text { Faktor 1: } \\
\text { Kritik und Mei- } \\
\text { nungsäußerung }\end{array}$ & $\begin{array}{l}\text { Faktor } 2 \text { : } \\
\text { Feedback und } \\
\text { neue Information }\end{array}$ & $\begin{array}{l}\text { Faktor 3: } \\
\text { Interesse } \\
\text { am Thema }\end{array}$ \\
\hline $\begin{array}{l}\text {... ich die Meinung von Journalisten } \\
\text { kritisieren kann }{ }^{\mathrm{a}}\end{array}$ & 3,05 & 0,790 & & \\
\hline $\begin{array}{l}\ldots \text { ich öffentlich Kritik an journalis- } \\
\text { tischen Inhalten üben kann }{ }^{\mathrm{a}}\end{array}$ & 3,30 & 0,764 & & \\
\hline $\begin{array}{l}\text {... ich meine Meinung öffentlich } \\
\text { äußern kann }{ }^{\mathrm{a}}\end{array}$ & 3,77 & 0,709 & & \\
\hline ... ich Spaß daran habe & 3,10 & $(0,464)$ & & 0,526 \\
\hline $\begin{array}{l}\text {... ich Journalisten so neue Infor- } \\
\text { mationen geben kann }{ }^{b}\end{array}$ & 2,91 & & 0,819 & \\
\hline $\begin{array}{l}\ldots \text { ich Korrekturvorschläge liefern } \\
\text { kann }^{\mathrm{b}}\end{array}$ & 2,97 & & 0,785 & \\
\hline $\begin{array}{l}\text {... ich Journalisten so Feedback } \\
\text { geben } \text { kann }^{\text {b }}\end{array}$ & 3,83 & & 0,699 & \\
\hline $\begin{array}{l}\text {... mich das Thema des Tweets } \\
\text { interessiert }\end{array}$ & 4,31 & & & 0,633 \\
\hline $\begin{array}{l}\ldots \text { ich Diskussionen zu öffentlich } \\
\text { relevanten Themen einleiten kann }\end{array}$ & 3,21 & & & 0,620 \\
\hline ... ich informiert werde & 3,18 & & & 0,790 \\
\hline ... ich Gleichgesinnte finden will & 2,40 & & & 0,488 \\
\hline Erklärte Varianz in \% & - & 20,3 & 18,7 & 18,7 \\
\hline
\end{tabular}

$n=106 ; 5$-Stufige Likert-Skala ( $1=$ trifft überhaupt nicht $\mathrm{zu}-5=$ trifft voll und ganz $\mathrm{zu})$

Faktorladungen $<0,350$ nicht dargestellt

Erklärte Gesamtvarianz: 57,6\%; KMO =0,731; Bartlett $=0,000$

Um die Interpretierbarkeit der Faktoren zu erleichtern, wurden nach einer ersten Hauptkomponentenanalyse die Items , ,... weil ich aktiv mitreden will“ $(M=3,88)$, ,... ich auf fehlende Standpunkte hinweisen kann“ $(M=3,70)$ und ,... ich in Kontakt mit Journalisten treten kann“ $(M=2,80)$ aufgrund von Querladungen $>0,400$ ausgeschlossen.

In der Tabelle sind die Ergebnisse der Faktorenanalyse zu den verbleibenden 11 Items dargestellt.

${ }^{a}$ Motiv-Skala „Kritik und Meinungsäußerung“ $(\alpha=0,71 ; M=3,37 ; S D=1,04)$. Das quer ladende Item „... ich Spaß daran habe“ wurde bei der Skalenbildung nicht berücksichtigt.

bMotiv-Skala ,Feedback und neue Informationen geben“ $(\alpha=0,72 ; M=3,24 ; S D=0,93)$

stark (1-3, 9-11)) gehen ebenfalls ein. ${ }^{3}$ Zusätzlich wird das politische Interesse der Befragten berücksichtigt. Zur Abbildung des Medienrepertoires der Befragten gehen häufig verwendete Informationsquellen (TV, Zeitungen, Blogs) ein. Darüber hinaus wird das generalisierte Vertrauen in Nachrichten einbezogen.

Die Ergebnisse der binär-logistischen Regression (Tab. 4) zeigen im Wesentlichen, dass Twitternde, die mit Journalisten häufig interagieren, eher starke als moderate politische Orientierungen aufweisen, d.h. eher zu den Polen des politischen

\footnotetext{
3 Es wird, Kalogeropoulos et al. (2017) folgend (siehe Abschn. 2), bei den starken Orientierungen nicht gesondert zwischen dem linken und dem rechten Pol in der Berechnung unterschieden. Im bivariaten Vergleich weisen Personen, die sich auf einer elfstufigen Skala politisch als stark rechts (= Skalenpunkte 9-11) einschätzen, signifikant häufiger Interaktionen mit Journalisten auf $\left(\chi^{2}(2)=7,678 ; p<0,05\right)$.
} 
Tab. 4 Determinanten einer häufigen Twitter-Interaktion mit Journalisten (Effektkoeffizienten einer binär-logistischen Regression, Befragung 2017)

\begin{tabular}{lcc}
\hline Variable & Exp (B) & {$[95 \%$ Konf.int.] } \\
\hline Geschlecht (Referenz: Mann) & 0,213 & {$[0,140 ; 3,298]$} \\
Frau & 1,026 & {$[0,967 ; 1,087]$} \\
Alter & & \\
Beschäftigung (Referenz: keine Beschäftigung) & 0,206 & {$[0,030 ; 1,405]$} \\
Beschäftigung ausgeübt & 2,540 & \\
Bildungsabschluss (Referenz: ohne Hochschulabschluss) & {$[0,496 ; 13,012]$} \\
Mit Hochschulabschluss & & \\
Politische Orientierung (Referenz: moderat) & $6,774^{*}$ & {$[1,335 ; 34,362]$} \\
Stark (zum linken oder rechten Pol) & 1,097 & {$[0,446 ; 2,698]$} \\
Persönlichkeitsstärke (Index) & 1,177 & {$[0,302 ; 4,578]$} \\
Politisches Interesse & 1,598 & {$[0,579 ; 4,409]$} \\
Politische Online-Partizipation (Index) & 1,041 & {$[0,253 ; 4,278]$} \\
Tägliche Zeitungsnutzung & 0,697 & {$[0,178 ; 2,728]$} \\
Tägliche TV-Nutzung & $4,805^{*}$ & {$[1,101 ; 20,959]$} \\
Mehrmals wöchentl. Blog-Nutzung & 0,677 & {$[0,313 ; 1,465]$} \\
Medienvertrauen & $2,465^{*}$ & {$[1,038 ; 5,854]$} \\
Motiv: Kritik und Meinungsäßßerung (Skala) & $4,975^{* *}$ & {$[1,728 ; 14,320]$} \\
Motiv: Feedback und neue Information (Skala) & 0,000 & - \\
Konstante & 0,575 & - \\
\hline Pseudo-R ${ }^{2}$ (Nagelkerke) & $44,837^{* * *}$ & - \\
Modell Chi-Quadrat & 79,1 & - \\
Prozentsatz richtig zugeordneter Fälle & & \\
\hline
\end{tabular}

$n=86$

$* * * p<0,001, * * p<0,01, * p<0,05$

Spektrums tendieren. Sie informieren sich zudem mit einer erhöhten Häufigkeit aus Blogs. Dies könnte einen verstärkten Gebrauch alternativer Informationsquellen implizieren. Unter den aus der Faktorenanalyse abgeleiteten Motivfaktoren erwiesen sich beide betrachteten Skalen als signifikante Determinanten $(p<0,05)$ des Vorliegens häufiger Interaktionen mit Journalisten auf Twitter. Besonders durch das Geben von „Kritik und Meinungsäußerung“ sowie durch „Feedback und neue Informationen“" motivierte Personen tendieren zu häufigen Twitter-Interaktionen. ${ }^{4}$

\section{Schlussfolgerungen und Einordnungen der Befunde}

Das politisch-journalistische Milieu wird in der politikwissenschaftlichen Literatur mit Metaphern wie ,Raumschiff“, ,Biotop“ oder ,Käseglocke“ umschrieben; es wird dadurch ein möglicher Mangel an Rückkopplung gegenüber den Bürgern an-

\footnotetext{
4 Wird statt einer binär-logistischen eine ordinal-logistische Regression testweise gerechnet, erweisen sich die Motivfaktoren ebenfalls als signifikante Determinanten (zur Begründung des Analyseverfahrens siehe Abschn. 3).
} 
genommen (z.B. Baugut 2017). Mit den in der Netzwerköffentlichkeit erweiterten Interaktionsmöglichkeiten scheinen die Verhältnisse zunehmend aufzubrechen. Gegenstand der vorliegenden Betrachtung sind die Bürger, die ihrerseits versuchen, Journalisten $\mathrm{zu}$ kontaktieren.

Die Nutzergruppe, die mit Journalisten auf Twitter interagiert, ist zahlenmäßig nicht so klein, wie vielleicht erwartet werden könnte, sollte die Plattform Twitter eher als ,exklusiver Tummelplatz“ für Medienakteure und Politiker betrachtet werden: Bei der Beantwortung von FF1 wurden mehrere zehntausend Accounts identifiziert, die Journalisten anschreiben und auf diese Weise eine Form der Online-Partizipation ausüben. Die Befragung ergab, dass es sich überwiegend um Nutzer mit einem hohen politischen Interesse handelt. Nicht nur Journalisten, sondern auch Politiker werden von den Befragten zu großen Teilen angeschrieben. In Folgestudien könnten die Muster dieser Anschriebe analog zur Nutzerkommentarforschung weiter untersucht werden, etwa mit Blick auf Inzivilität oder auch Manipulationsversuche, die auf Twitter verstärkt zu beobachten sind (Thieltges und Hegelich 2017).

Hinsichtlich der Motivation der journalistischen Interaktionspartner kristallisieren sich mehrere Motivfaktoren heraus. Ein Teil der Befragten scheint vor allem das Bedürfnis nach klarer Meinungsäußerung und Kritik zu hegen, während ein anderer Teil Feedbackmotive wie etwa das Weitergeben von neuen Informationen an Journalisten äußert. Etwas überspitzt kann man darin Hinweise auf die anfangs skizzierten hellen und dunklen Seiten der Partizipation erkennen (Quandt 2018). Nutzer, die ihre Meinungen zu Tweets und Berichten von Journalisten verfassen, beeinflussen so auch die Dynamik und die Gestalt politischer Diskurse in einem hybriden Mediensystem mit (Jungherr et al. 2019). Interessanterweise erwiesen sich beide genannten Motivfaktoren mit einem häufigen Schreiben von Tweets an Journalisten assoziiert. Für den Journalismus lässt sich hieraus wiederum ableiten, dass die untersuchte Form der Nutzerbeteiligung insgesamt einer differenziert-kritischen Beurteilung bedarf. Dass die Befragten Beschimpfungen von Journalisten durch andere Nutzer wahrnehmen sowie der Befund, dass eher Personen mit starken politischen Orientierungen häufiger Journalisten in ihren Tweets anschreiben, zeigt, dass es sich insgesamt um kein friedvolles Kommunikationsumfeld handelt.

Aus den weiteren Befunden dieser Studie kann geschlussfolgert werden, dass es sich bei den Befragten um eine Sondergruppe der ,aktiven Twitterer“ handelt. Die „aktiven Twitterer“ unterscheiden sich von durchschnittlichen Internetnutzern in demographischer Hinsicht, mit Blick auf ihre Interessen und ihre Persönlichkeitsstärke (Hölig 2018). Es überrascht nicht, dass jene Merkmale bei den hier untersuchten Twitternden die Häufigkeit des Austauschs mit Journalisten nicht weitergehend erklären. Wird hingegen gefragt, wodurch sich die Anschreibenden allgemein von Nutzern unterscheiden, die sich nicht an Journalisten wenden, werden vermutlich die von Hölig herausgehobenen Merkmale relevant sein: Es handelt sich um Personen mit einem höheren Interesse an Nachrichten und Politik, zum anderen wahrscheinlich um eine Gruppe, ,die tendenziell meinungsstärker und von sich überzeugter ist als die durchschnittliche Bevölkerung“ (Hölig 2018, S. 159). Es ist davon auszugehen, dass das raue Diskursklima auch damit zusammenhängt. Die genauen Unterschiede zwischen den Anschreibenden und der Durchschnittsbevölkerung wären allerdings in Folgestudien zu erhellen. Gleiches gilt für die möglicherweise negative Motivati- 
on, die bei einem Teil der Twitternden in ihrer ausgeprägten Neigung zu kritisieren, durchscheint. Hier müssten Faktoren, die z. B. Hassrede gegenüber Journalisten oder Politikern begünstigen, noch näher beleuchtet werden.

Das Tracking hat ergeben, dass sich die Anschriebe sehr ungleich auf die untersuchten Politikjournalisten verteilen, das heißt, dass nur wenige von ihnen sehr viel Resonanz erfahren. Eine Befragung von Journalisten und Politikern speziell zu ihren Interaktionserfahrungen mit Bürgern auf Twitter würde ebenfalls eine wichtige Ergänzung zur Einordnung der vorgelegten Befunde darstellen. Auch eine ländervergleichende Betrachtung ist in diesem Zusammenhang ein Desiderat. So wird Twitter zum Beispiel in Großbritannien und den USA sowohl in der Bevölkerung als auch unter Journalisten deutlich häufiger verwendet als es aktuell in Deutschland der Fall ist (siehe im Überblick Nuernbergk 2018). Die Beobachtungen von Chadwick (2013) zur Herausbildung eines hybriden Mediensystems betonen wiederholt eine zentrale Rolle von Twitter. Dass sich diese Beispiele zuerst auf die USA und Großbritannien bezogen haben, ist daher kein Zufall.

Eine wesentliche Limitation der vorliegenden Studie ist die niedrige Teilnehmerzahl. Mit den vorhandenen Ressourcen war es lediglich möglich, eine Stichprobe von 4000 Nutzern anzuschreiben. Ob daraus alle betreffenden Nutzer die Survey-Einladung tatsächlich gesehen haben, kann nicht mit Sicherheit nachvollzogen werden, da bei @-Mentions keine Zustellbestätigungen erfolgen. In dieser Studie konnten auch nur Accounts erfasst werden, die sich in öffentlicher Form beteiligen. Trotz der über das Tracking ermöglichten Identifikation derjenigen Accounts, die Journalisten anschreiben, sind die Optionen, die dahinter stehenden Nutzer anschließend zu ihren Einstellungen zu befragen, technisch erschwert; ein Massenversand weitgehend identischer Einladungstweets wird auf Twitter folglich unterbunden. ${ }^{5}$ Dies könnte ein Grund dafür sein, warum dieser Weg bislang im Rahmen von Befragungen kaum praktiziert wurde. Eine Alternative könnten wiederum Online-Access-Panels darstellen. Fraglich ist jedoch, ob dadurch tatsächlich eine ausreichende Zahl von Nutzern rekrutiert werden kann, die mit Journalisten auf Twitter interagieren.

Die hier vorgelegten Ergebnisse dokumentieren, dass einige Akteure im politischmedialen Komplex unter intensiver Beobachtung durch ein vernetztes Twitter-Publikum stehen; diese Beobachtungen artikulieren sich wiederum wenigstens zum Teil durch zehntausende Rückmeldungen in Form von Anschrieben. Auf Twitter findet auch auf diese Weise ein dynamischer und affektiver Wettstreit um Deutungen statt, bei dem oft kritische, emotionale und nicht-rationale Formen der Beteiligung sichtbar werden (Pfetsch et al. 2018). Die untersuchten Beteiligungsformen scheinen folgenreicher zu sein als in anderen Kommunikationsräumen im Internet, da sie wenn sie einmal Dynamik gewinnen - rasch Politiker, Journalisten und Sprecher erreichen und dort nicht selten zu Anschlusskommunikation führen.

Danksagung Für ihre Hinweise zur Überarbeitung des Manuskripts danke ich beiden anonymen Gutachter/innen und den Herausgeber/innen sowie Peter Maurer und Nina Fabiola Schumacher. Axel Bruns danke ich für unsere Kooperation beim Tracking von Journalist/innen.

\footnotetext{
5 „Malicious behavior detection systems“ auf Twitter identifizieren ein solches Verhalten als verdächtig, insbesondere dann, wenn auf die @ -Erwähnungen keine Antworten der angeschriebenen Nutzer erfolgen. Insofern müssen Einladungen kleinschrittig versendet werden, was den Aufwand erhöht.
} 
Förderung Unterstützt durch Australian Research Council (DP160101211 Journalism beyond the Crisis: Emerging Forms, Practices and Uses).

Funding Open Access funding provided by Projekt DEAL.

Open Access Dieser Artikel wird unter der Creative Commons Namensnennung 4.0 International Lizenz veröffentlicht, welche die Nutzung, Vervielfältigung, Bearbeitung, Verbreitung und Wiedergabe in jeglichem Medium und Format erlaubt, sofern Sie den/die ursprünglichen Autor(en) und die Quelle ordnungsgemäß nennen, einen Link zur Creative Commons Lizenz beifügen und angeben, ob Änderungen vorgenommen wurden.

Die in diesem Artikel enthaltenen Bilder und sonstiges Drittmaterial unterliegen ebenfalls der genannten Creative Commons Lizenz, sofern sich aus der Abbildungslegende nichts anderes ergibt. Sofern das betreffende Material nicht unter der genannten Creative Commons Lizenz steht und die betreffende Handlung nicht nach gesetzlichen Vorschriften erlaubt ist, ist für die oben aufgeführten Weiterverwendungen des Materials die Einwilligung des jeweiligen Rechteinhabers einzuholen.

Weitere Details zur Lizenz entnehmen Sie bitte der Lizenzinformation auf http://creativecommons.org/ licenses/by/4.0/deed.de.

\section{Literatur}

Albæk, Erik, Arjen van Dalen, Nael Jebril, und Claes H. de Vreese. 2014. Political journalism in comparative perspective. New York: Cambridge University Press.

Baugut, Philip. 2017. Politiker und Journalisten in Interaktion. Einfluss des Medienwettbewerbs auf lokale politische Kommunikationskulturen. Wiesbaden: Springer VS.

Binns, Amy. 2017. Fair game? Journalists' experiences of online abuse. Journal of Applied Journalism \& Media Studies 6(2):183-206.

Borra, Erik, und Bernhard Rieder. 2014. Programmed method: Developing a toolset for capturing and analyzing tweets. Aslib Journal of Information Management 66(3):262-278.

Borucki, Isabelle, und Uwe Jun. 2018. Regierungskommunikation im Wandel - Politikwissenschaftliche Perspektiven. In Regierungskommunikation und staatliche Öffentlichkeitsarbeit, Hrsg. Juliana Raupp, Jan Niklas Kocks, und Kim Murphy, 24-46. Wiesbaden: VS.

Broersma, Marcel, und Todd Graham. 2016. Tipping the balance of power. In The Routledge companion to social media and politics, Hrsg. Axel Bruns, Gunn Enli, Eli Skogerbø, Anders Olof Larsson, und Christian Christensen, 89-103. London: Routledge.

Bruns, Axel. 2018. Gatewatching and news curation: Journalism, social media, and the public sphere. New York: Peter Lang.

Bruns, Axel, und Moe Hallvard. 2014. Structural layers of communication on Twitter. In Twitter and society, Hrsg. Katrin Weller, Axel Bruns, Jean Burgess, Merja Mahrt, und Cornelius Puschmann, 15-28. New York: Peter Lang.

Bruns, Axel, und Christian Nuernbergk. 2019. Political journalists and their social media audiences: new power relations. Media and Communication 7(1):198-212.

Chadwick, Andrew. 2013. The hybrid media system: politics and power. New York: Oxford University Press.

Dohle, Marco, Olaf Jandura, und Gerhard Vowe. 2014. Politische Kommunikation in der Online-Welt. Dimensionen des strukturellen Wandels politischer Kommunikation. ZfP Zeitschrift für Politik 61(4):414-436.

Fletcher, Richard, und Sora Park. 2017. The impact of trust in the news media on online news consumption and participation. Digital Journalism 5(10):1281-1299.

Frees, Beate, und Wolfgang Koch. 2018. ARD/ZDF-Onlinestudie 2018: Zuwachs bei medialer Internetnutzung und Kommunikation. Media Perspektiven 7(8):398-413.

Friemel, Thomas N., und Mareike Dötsch. 2015. Online reader comments as indicator for perceived public opinion. In Kommunikationspolitik für die digitale Gesellschaft Digital communication research, Bd. 1, Hrsg. Martin Emmer, Christian Strippel, 151-172. https://doi.org/10.17174/dcr.v1.0.

GESIS. 2010. Allgemeine Bevölkerungsumfrage der Sozialwissenschaften ALLBUS 2008, GESIS Datenarchiv, Köln, ZA4600 Datenfile Version 1.0.0. https://doi.org/10.4232/1.4600. 
Gil de Zúñiga, Homero, Jung Nakwon, und Sebastián Valenzuela. 2012. Social media use for news and individuals' social capital, civic engagement and political participation. Journal of Computer-Mediated Communication 17(3):319-336.

Greck, Regina. 2017. Elitäre Verhältnisse. Selbst- und Fremdbild der Eliten in Journalismus und Politik. Wiesbaden: Springer VS.

Hanusch, Folker, und Daniel Nölleke. 2019. Journalistic homophily on social media. Digital Journalism 7(1):22-44.

Hoffmann, Christian. 2016. Lügenpresse!!!!, EJO. http://de.ejo-online.eu/qualitaetethik/luegenpresse. Zugegriffen: 10. Febr. 2017.

Hölig, Sascha. 2018. Eine meinungsstarke Minderheit als Stimmungsbarometer?! Über die Persönlichkeitseigenschaften aktiver Twitterer. Medien \& Kommunikationswissenschaft 66(2):140-169.

Hölig, Sascha, und Uwe Hasebrink. 2016. Reuters Institute Digital News Survey 2016: Ergebnisse für Deutschland. Arbeitspapiere des Hans-Bredow-Instituts 38. https://hans-bredow-institut.de/uploads/ media/Publikationen/cms/media/3ea6d4fed04865d10ad27b3f98c326d3a0ae6c29.pdf. Zugegriffen: 9. Juni 2020.

Hölig, Sascha, und Uwe Hasebrink. 2018. Reuters Institute Digital News Report 2018: Ergebnisse für Deutschland. Arbeitspapiere des Hans-Bredow-Instituts 44. https://hans-bredow-institut.de/uploads/ media/Publikationen/cms/media/t611qnd44RDNR18_Deutschland.pdf. Zugegriffen: 23. Juni 2019.

Jouët, Josiane. 2018. Revisiting digital news audiences with a political magnifying glass. Javnost-The Public 25(1-2):119-126.

Jungherr, Andreas, Oliver Posegga, und An Jisun. 2019. Discursive power in contemporary media systems: a comparative framework. The International Journal of Press/Politics 24(4):404-425.

Kalogeropoulos, Antonis, Samuel Negredo, Ike Picone, und Rasmus Kleis Nielsen. 2017. Who shares and comments on news? A cross-national comparative analysis of online and social media participation. Social Media + Society 3(4):1-12.

Katz, Elihu, Jay G. Blumler, und Michael Gurevitch. 1973. Uses and gratifications research. The Public Opinion Quarterly 37(4):509-523.

Kümpel, Anna Sophie, Veronika Karnowski, und Till Keyling. 2015. News sharing in social media: a review of current research on news sharing users, content, and networks. Social Media + Society $1(2): 1-14$.

Lobigs, Frank, und Christoph Neuberger. 2018. Meinungsmacht im Internet und die Digitalstrategien von Medienunternehmen: neue Machtverhältnisse trotz expandierender Internet-Geschäfte der traditionellen Massenmedien-Konzerne. Berlin: Vistas.

Maurer, Peter, und Markus Beiler. 2018. Networking and political alignment as strategies to control the news. Journalism Studies 19(14):2024-2041.

Neuberger, Christoph. 2018. Journalismus in der Netzwerköffentlichkeit. Zum Verhältnis zwischen Profession, Partizipation und Technik. In Journalismus im Internet, Hrsg. Christian Nuernbergk, Christoph Neuberger, 11-80. Wiesbaden: Springer VS.

Neuberger, Christoph, Susanne Langenohl, und Christian Nuernbergk. 2014. Social Media und Journalismus. Düsseldorf: Landesanstalt für Medien Nordrhein-Westfalen.

Noelle-Neumann, Elisabeth. 1986. Der selbständige Mensch. Ein theoretisches Konzept Schelskys wird empirisch mit einer „Skala der Persönlichkeitsstärke“ untersucht. In Helmut Schelsky - ein Soziologe in der Bundesrepublik. Eine Gedächtnisschrift von Freunden, Kollegen und Schülern, Hrsg. Horst Baier, 182-194. Stuttgart: Enke.

Nuernbergk, Christian. 2016. Political journalists' interaction networks: The German Federal Press Conference on Twitter. Journalism Practice 10(7):868-879.

Nuernbergk, Christian. 2018. Recherche im Internet. In Journalismus im Internet: Profession - Partizipation - Technisierung, Hrsg. Christian Nuernbergk, Christoph Neuberger, 101-138. Wiesbaden: Springer VS.

Parmelee, John H. 2014. The agenda-building function of political tweets. New Media \& Society 16(3):434-450.

Pfetsch, Barbara, und Eva Mayerhöffer. 2011. Vordergründige Nähe. Zur Kommunikationskultur von Politik- und Medieneliten in Deutschland. Medien \& Kommunikationswissenschaft 59(1):40-59.

Pfetsch, Barbara, Maria Löblich, und Christiane Eilders. 2018. Dissonante Öffentlichkeiten als Perspektive kommunikationswissenschaftlicher Theoriebildung. Publizistik 63(4):477-495.

Preuß, Marlene, Frederik Tetzlaff, und Andreas Zick. 2017. „Publizieren wird zur Mutprobe“. Studie zur Wahrnehmung und Erfahrungen mit Angriffen unter Journalist_innen, Mediendienst Integration, Bielefeld. https://mediendienst-integration.de/fileadmin/Dateien/Studie-hatespeech.pdf. Zugegriffen: 23. Juni 2019. 
Quandt, Thorsten. 2018. Dark participation. Media and Communication 6(4):36-48.

Reschke, Anja. 2016. Aber was heißt denn das: „Sagen, was ist“?, Übermedien, Berlin. http://uebermedien. de/1995/aber-heisstdenn-das-sagen-ist/. Zugegriffen: 18. Jan. 2019.

Schatto-Eckrodt, Tim, Svenja Boberg, Florian Wintterlin, Lena Frischlich, und Thorsten Quandt. 2019. Bedrohte Deliberation: Information Warfare und Desinformation als Bedrohung digitaler Öffentlichkeiten. Communicatio Socialis 52(2):147-158.

Schenk, Michael, und Ljewin Scheiko. 2011. Meinungsführer als Innovatoren und frühe Übernehmer des Web 2.0. Media Perspektiven 9:423-431.

Schindler, Johanna, Claudia Fortkord, Lone Posthumus, Magdalena Obermaier, Nayla Fawzi, und Carsten Reinemann. 2018. Woher kommt und wozu führt Medienfeindlichkeit? Zum Zusammenhang von populistischen Einstellungen, Medienfeindlichkeit, negativen Emotionen und Partizipation. Medien \& Kommunikationswissenschaft 18(3):283-301.

Schmidt, Jan-Hinrik. 2014. Twitter and the rise of personal publics. In Twitter and society, Hrsg. Katrin Weller, Axel Bruns, Jean Burgess, Merja Mahrt, und Cornelius Puschmann, 3-14. New York: Peter Lang.

Schultz, Tanjev, Nikolaus Jackob, Marc Ziegele, Oliver Quiring, und Christian Schemer. 2017. Erosion des Vertrauens zwischen Medien und Publikum. Media Perspektiven 5:246-259.

Springer, Nina. 2011. Suche Meinung, biete Dialog? Warum Leser die Kommentarfunktion auf Nachrichtenportalen nutzen. In Medieninnovationen, Hrsg. Jens Wolling, Andreas Will, und Christina Schumann, 247-264. Konstanz: UVK.

Springer, Nina, und Anna Sophie Kümpel. 2018. User-Generated (Dis)Content. In Journalismus im Internet, 2. Aufl., Hrsg. Christian Nuernbergk, Christoph Neuberger, 241-271. Wiesbaden: Springer VS.

Springer, Nina, Ines Engelmann, und Christian Pfaffinger. 2015. User comments: motives and inhibitors to write and read. Information, Communication \& Society 18(7):798-815.

Thieltges, Andree, und Simon Hegelich. 2017. Manipulation in sozialen Netzwerken. ZfP Zeitschrift für Politik 64(4):493-512.

Thimm, Caja, Jessica Einspänner, und Mark Dang-Anh. 2012. Twitter als Wahlkampfmedium. Publizistik 57(3):293-313.

Valenzuela, Sebastián, Kim Yonghwan, und Homero Gil de Zúñiga. 2012. Social networks that matter: Exploring the role of political discussion for online political participation. International Journal of Public Opinion Research 24:163-184.

Vonbun, Ramona, und Klaus Schönbach. 2014. Wer ist politisch aktiv im Social Web? Publizistik 59(2):199-212.

Ziegele, Marc. 2016. Nutzerkommentare als Anschlusskommunikation: Theorie und qualitative Analyse des Diskussionswerts von Online-Nachrichten. Wiesbaden: Springer VS.

Ziegele, Marc, Marius Johnen, Andreas Bickler, Ilka Jakobs, Till Setzer, und Alexandra Schnauber. 2013. Männlich, rüstig, kommentiert? Einflussfaktoren auf die Aktivität kommentierender Nutzer von Online-Nachrichtenseiten. Studies in Communication | Media 2(1):67-114. 